\title{
SOME DIFFERENTIAL GAMAS WITH INCOMPLETE INFORMATION
}

F.I. Chernousko, A.A. Melikyan

Institute for Probiems of Mechanics

of the USSR Academy of Sciences

Moscow, USSR

This paper deals with some classes of differential games in which one player has no complete information about the phase coordinates of his partner. The information conditions under consideration allow information delay and $(o r)$ gaps of information. The equivalence of these games with incomplete information to certain games with complete information is shown. The solutions of a number of special problems of differential games are given.

I. Formulation of the problem. Let the motion of two controlled objects (players) $X$ and $Y$ on fixed time interval[to, $T]$ be described by differential equations and constraints

$$
\begin{array}{cc}
X: \dot{x}=f(t, x, u), & Y: \dot{y}=g(t, y, v), \\
u \in U, & v \in V .
\end{array}
$$

The dimensions of phase-vectors $x, y$ and control-vectors $u, v$ are arbitrary; the vector-functions $f, g$ are given; $U, V$ are bounded closed sets in spaces of vectors $u, v$ respectively. Dots denote the time derivatives.

The information conditions of $X$ are the following. Let $\theta=$ $\theta(t)$ be a continuously differentiable scalar function on $\left[t_{0}, T\right]$, such that $\theta(t) \leqslant t, t \in\left[t_{0}, T\right]$. We assume that the following set of quantities becomes known by the player $X$ at every moment $t \in\left[t_{0}, T\right]$ : $w=\{t, x(t), y(\theta(t))\}$; i.e. $X$ knows precisely his own phase-vector at every moment of time and receives information about phase-vector of $Y$ with variable time delay $t-\theta(t) \geqslant 0$.

The initial data for (I.I) are of the form

$$
x\left(t_{0}\right)=x^{0}, \quad y\left(\theta\left(t_{0}\right)\right)=y^{0} .
$$


The player $X$ chooses his control vector $u$ at a moment $t \in\left[t_{0}, T\right]$, knowing the information which he obtains at that moment, i.e. he applies feed-back (closed-loop) control strategies of the form $U=$ $u(w)$

The aim of $X$ is to minimize the functional

$$
J=F(x(T), y(T)) \text {, }
$$

where $F$ is a given function. The player $Y$ counteracts

and realizes his control in the form of time functions $V(t)$. It is clear that control $V(t)$ must be given on the interval $\left[\theta\left(t_{0}\right), T\right]$.

Thus, if $X$ and $Y$ choose certain fixed strategy $\hat{u}(w)$ and control $\hat{v}(t)$, then the value $J(\hat{u}, \hat{v})$ can be calculated, in principle, in the following way. The time function $\hat{V}(t)$ can be substituted in the second equation ( $I . I)$ and this equation can be integrated on $\left[\theta\left(t_{0}\right), T\right]$ with initial condition (I.2). Substituting the function $y(\theta(t))$ in the strategy $\hat{U}(w)$ and, further, $\hat{U}(w)$ in the first equation (I.I), one can obtain the trajectory $x(t), t \epsilon$ $\left[t_{0}, T\right]$, with initial point (I.2). Hence, the values $y(T), x(T)$ and, therefore, the value of functional (I.3) are known. Note, that to construct the trajectory of $X$ on $\left[t_{0}, T\right]$ one needs the trajectory of $Y$ on interval $\left[\theta\left(t_{0}\right), \theta(T)\right]$.

We consider only such functions $U(w), V(t)$, which define a unique absolutely continuous solution of (I.I). Considering the game (I.I)-(I.3) from the point of view of $X$, we state the following problem of differential game.

Problem I. Find the optimal guaranteeing strategy $U^{*}$ of the player $X$, i.e. the minimax strategy, which satisfies the equality

$$
J^{*}=\min _{u} \sup _{v} J(u, v)=\sup _{v} J\left(u^{*}, v\right) \text {, }
$$

where min and sup are taken among the above described strategies $u(w)$ and control functions $V(t)$. The information conditions of $Y$ are not essential for problem $I$.

2. The equivalent game with complete information. Let us show now that the problem $I$ is equivalent to some game with standard information conditions. Consider the dynamic equation of $Y$, corresponding 
to some control $V(t), t \in\left[\theta\left(t_{0}\right), T\right]$. Introduce the notation: $Z(t)=$ $y(\theta(t)), t \in\left[t_{0}, T\right]$. For the derivative of vector-function $Z(t)$ we have the equality $\dot{z}=\dot{\theta} d y / d \theta$, which we can write by means of equation ( $I . I$ ) in the form

$$
\dot{z}=\dot{\theta} \cdot g(\theta(t), z(t), \tilde{v}(t)),
$$

where $\tilde{V}(t)$ denotes $v(\theta(t))$. The functional (I.3) for the new game is constructed in the following way. Obtaining at the moment $T$ the information about the phase-vector $y(\theta(T))=Z(T)$, the player $X$ can conclude that the phase-vector $y(T)$ belongs to domain $G(Z(T)$, $\theta(T), T)$. Here $G(z, t, T)$ denotes the domain attainable at the moment $T$ for the object $Y$ from ( $I . I)$, provided that at the moment $t$ his phase-vector is equal to $Z$. We define now the functional

$$
\begin{aligned}
\tilde{J}=\tilde{F}(x(T), z(T))= & \max F(x(T), \eta) . \\
& \eta \in G(z(T), \theta(T), T)
\end{aligned}
$$

We introduce a new game, defined by relations

$$
\begin{gathered}
X: \dot{x}=f(t, x, u), \quad Y: \dot{z}=\dot{\theta} \cdot g(\theta(t), z, \tilde{v}), \\
t \in\left[t_{0}, T\right], x\left(t_{0}\right)=x^{0}, u \in U, \quad z\left(t_{0}\right)=y^{0}, \tilde{v} \in V,
\end{gathered}
$$

and by the functional (2.2). The player $X$ in this game can observe set of quantities $\tilde{w}=\{t, x(t), z(t)\}$ at each moment $t \in\left[t_{0}, T\right]$ and applies strategies of the form $U=U(\tilde{W})$. The problem of finding the optimal guaranteeing strategy in the game $(2.3),(2.2)$ with complete information is equivalent to the original problem $I$. This statement is evident from the previous constructions. Both games have the same optimal value of the minimized functional. For the particular cases of function $\theta(t)$ these problems were considered earlier in the papers $[\mathrm{I}-5]$.

If we take $\theta(t)=t-\tau, \tau=$ const., we obtain differential games with constant delay of information. The equivalence of such games to certain games with complete information was shown in the paper [I]. In the paper [2] some examples for $\theta(t)=t-\tau$ were considered.

Papers [3-5] are devoted to the games in which the player $X$ observes his partner's phase-vector only on time intervals $\left[a_{i}, b_{i}\right]$, $i=1, \ldots, N, a_{1}=t_{0}, b_{N}=T, b_{i}<a_{i+1}, i=1, \ldots, N-1$. These 
games can be considered as a limiting case of the games of section I with the following $\theta(t)$ :

$$
\begin{aligned}
& \theta(t)=t, \quad t \in\left[a_{i}, b_{i}\right], \quad i=1, \ldots, N, \\
& \theta(t)=b_{i}, \quad t \in\left(b_{i}, a_{i+1}\right), i=1, \ldots, N-1 .
\end{aligned}
$$

The intervals $\left[a_{i}, b_{i}\right]$ can be either fixed or chosen by the first player (the problem of optimal observations) or chosen by the second player (the problem of optimal information noise). In the last two cases it is natural to constrain the number or the total time of observations or noises (see ref. [3-5]). Some examples of the problems of these types are given below.

3. Examples. a) Let the relations (I.I) on the interval $[0, T]$ for objects $X$ and $Y$ of the same type have one of two forms

$$
\begin{array}{ll}
\dot{x}=u, x(0)=x^{0}, & \dot{y}=v, y(0)=y^{0}, \\
\ddot{x}=u,|u| \leqslant \mu, & \ddot{y}=v,|v| \leqslant \nu \\
x(0)=x^{0}, \dot{x}(0)=\xi^{\circ}, & y(0)=y^{0}, \dot{y}(0)=\eta^{\circ}, \mu>\nu>0,
\end{array}
$$

i.e. both objects are controlled either by their velocities or by their accelerations. We consider the set of observation moments to consist of $N$ discrete moments of observations $a_{i}: t_{0}=a_{1} \leqslant a_{2} \leqslant$ $\ldots \leqslant a_{N}=T$. The functional (I.3) is of the form

$$
\mathcal{J}=|x(T)-y(T)|
$$

We consider the problem of finding the distribution of moments $a_{i}^{*}$ which is optimal for the player $X$ (in the sense of minimum of (3.3)). The equivalent game with complete information in this case is a multistep game, and it can be solved analytically by means of dynamic programming (see ref. [3], [4]). As the result we obtain the following formulas for optimal moments of observations in the games (3.I), (3.2) respectively

$$
\begin{aligned}
& a_{i}^{*}=T \cdot \mathcal{L}_{N}(k, i), \quad \mathcal{L}_{N}(k, i)=\left(1-k^{i-1}\right) /\left(1-k^{N-1}\right), \\
& a_{i}^{*}=T \sqrt{\mathcal{L}_{N}(k, i)}, \quad k=\nu / M<1, i=1, \ldots, N .
\end{aligned}
$$

The formulas for the minimal value of functional (3.3) guaranteed for the player $X$ also were obtained. For instance, in the game (3.I) we have 


$$
T^{*}=\max \left[\left|x^{0}-y^{0}\right|-(\mu-\nu) T, \mu T k^{N-1}(1-k) /\left(1-k^{N-1}\right)\right] \text {. }
$$

b) Definition. A set $Q$ of moments of observations belonging to the interval $\left[t_{0}, T\right]$ is called a sufficient observation set for a given inftial position, if the observations on $Q$ guarantee for the player $X$ the same result (in the sense of the functional (I.3)) as the continuous observations on the interval $\left[t_{0}, T\right]$.

The explicit formulas obtained permit us to find in the problems (3.I), (3.2) the minimal sufficient observation sets. For some initial positions these sets consist of a finite number of observation moments. Note, that a denumerable set of moments of observations $a_{i}^{*}$, $i=1,2, \ldots$, obtained from (3.4) by setting $N \rightarrow \infty$, with a point of condensation at $t=T$ is a sufficient set for all initial positions.

c) We assume now, that the terminal moment $T$ in the games (3.I), (3.2) is not fixed and is defined by the following capture condition: $|x(T)-y(T)| \leqslant l, l>0$. The player $X$ desires to minimize the time of pursuit $T$. It can be shown that observing the object $Y$ at a finite number of moments the player $X$ can capture $Y$ during the same optimal time as in the corresponding games with information. The minimal sufficient number of moments of observations for (3.I) depends on initial position as follows

$$
N\left(x^{\circ}, y^{\circ}\right)=1-\left[\left(\ln \left|x^{\circ}-y^{\circ}\right|-\ln l\right) / \ln k\right] \text {, }
$$

where the square brackets denote the greatest integer. A similar formula holds for the game (3.2) (see ref. [4]).

d) Let the motion of players $X$ and $Y$ during the fixed time interval $[0, T]$ be described by the following equations

$$
\ddot{x}=u, \quad|u| \leqslant 1, \quad \dot{y}=v, \quad|v| \leqslant 1,
$$

and the player $X$ minimize the functional (3.3). Using the technique of equivalent games, one can show, that a sufficient observation set for all initial positions has a unique point of condensation which is equal to $t=T-1$ when $T>1$ and to $t=0$, when $T \leqslant 1$. Considering the game from the point of view of maximizing player $\dot{Y}$, we find out that to guarantee for $Y$ the maximum value of functional (3.3) it is necessary and sufficient to observe the position of $X$ 
at two moments of time: 0 and $T-1 \quad(T>1)$ (see ref. [5]).

$Y e)$ Let the set of moments of observations be chosen by the player $Y$. It means that the player $Y$ can switch on the noise which eliminates the observations made by the player $X$. The problem is to find the optimal distribution of intervals of noise $\left(b_{i}, a_{i+1}\right), i=1$,. $\ldots, N-1$, during which the observations for $X$ are impossible. The total duration of these intervals is equal to $\vartheta$. A number of specific problems of that type was considered. In these problems it occurred that the optimal set of noise intervals consists only of one interval of the total length $\mathcal{V}$, i.e. the noise is concentrated in one time interval. In different cases this interval is situated at the beginning, at the end of or inside the interval of motion.

f) Consider an example from ref. [2] with $\theta(t)=t-\tau, \tau=$ const., i.e. the player $X$ receives the information about his partner's phase vector with constant delay $\tau$. Let the motion of players be described by the equation and limitations (3.2) and the game be finished as soon as the condition $|x(T)-y(T)| \leqslant l$ holds. The player $X$ desires to minimize the time of pursuit $T$. Using the technique of section $I$, it was shown that the capture is available for $X$ from any initial position if the inequality $(\mu-\nu) / \mu>\nu \tau^{2} /(2 \cdot l)$ holds. Putting $\tau=0$ we obtain the well-known condition of capture $\mu>\nu$ for the game with complete information. A number of other examples can be found in the references given below.

\section{REFHRENCES}

I. Chernousko, F.I. On differential games with delay of information. (Russian) Dokl. Akad. Nauk SSSR I88 (I969), 766-769.

2. Sokolov, B.N., Chernousko, F.I. Differential games with delay of information. (Russian) Prikl. Hat. i Mekh. 34 (I970), no.5,8I2-8I9.

3. Melikyan, A.A., Chernousko, F.I. On differential games with alternating conditions of information. (Russian) Dokl. Akad. Nauk SSSR 203 (I972), 46-49.

4. Melikyan, A. A. On differential games with information gaps. (Russian. Armenian and English summaries) Isv. Akad. Nauk Arm. SSR, Matematika I972, no.4, 300-308.

5. Melikyan, A.A. On minimal observations in one game of approaching. (Russian) Prikl. Mato i Mekh. 37 (I973), no.3, 426-433. 